\title{
Long Bubble Penetration through Viscoelastic Fluids in a Suddenly Contracting and Expanding Tube
}

\author{
Ching-Chuan Chang, ${ }^{1}$ Huang-Chih Lin, ${ }^{2}$ Ming-Yuan Lin, ${ }^{1}$ and Te-Hui Tsai ${ }^{1}$ \\ ${ }^{1}$ Department of Mechanical Engineering, Army Academy R.O.C., Taoyuan 320, Taiwan \\ ${ }^{2}$ Department of Aircraft Engineering, Army Academy R.O.C., Taoyuan 320, Taiwan \\ Correspondence should be addressed to Te-Hui Tsai; tsai57092001@yahoo.com.tw
}

Received 24 May 2016; Revised 13 July 2016; Accepted 20 July 2016

Academic Editor: Ying Li

Copyright (c) 2016 Ching-Chuan Chang et al. This is an open access article distributed under the Creative Commons Attribution License, which permits unrestricted use, distribution, and reproduction in any medium, provided the original work is properly cited.

This study investigated the properties of long bubbles penetrating viscoelastic fluids in a suddenly contracting and expanding tube. Injection gas flow is controlled by a mass flow controller (MFC). Some of dimensionless parameters, such as the capillary number $(\mathrm{Ca})$, the Reynolds number $(\mathrm{Re})$, the fractional ratio $(m)$, and the Weissenberg number (Wi), are discussed herein. The experimental results showed that bubble velocity, $\mathrm{Ca}$, and $\mathrm{Wi}$ increase as shear viscosity increases under a constant gas flow by MFC. However, as shear viscosity increases, bubble diameter decreases, and $m$ increases. When gas flow is $200 \mathrm{~mL} / \mathrm{min}$ and shear viscosity increases, the bubble front is sharper in the contraction tube, and the bubble front shape is blunter in the sudden expansion tube. When gas flow is $600 \mathrm{~mL} / \mathrm{min}$ and shear viscosity increases, the bubble front is blunter in the contraction tube and exhibits a torch shape in the sudden expansion tube.

\section{Introduction}

Gas penetration through a viscoelastic fluid depends on conditions such as the film coating on the inner wall of a tube, displacement of oil by a low-viscosity fluid in enhanced oil recovery, and fluid replacement in gas-assisted injection molding (GAIM). Many industrial applications involve gas penetration through viscoelastic fluids. For example, gas penetrates viscoelastic fluids in GAIM. When using GAIM technique for manufacturing products with hollow structures, a compressed gas such as nitrogen is injected into the polymer melt core of a mold during the usual injection molding process. The interface between the gas and the polymer melt moves to replace the polymer melt with the gas. Because polymer melts are viscoelastic fluids, the viscoelastic properties of polymer melts affect interface motion. The GAIM avoids the shortcomings of traditional methods, such as surface shrinkage and irregular work piece thickness. The GAIM is also widely used to manufacture components for automobiles, home appliances, communication products, computer chasses, and other items.
Early studies of gas penetration in a tube analyzed Newtonian fluids [1-15]. The relationship between fractional ratio and capillary number in Newtonian fluids can be described by a master curve. Many engineering polymers used in polymer processing have shear-thinning viscosity, which is an important factor in polymer processing flow [6]. Thus, the effect of shear-thinning viscosity on bubble-tip behavior needs clarification. Yang and Chou [16] examined gas penetration through a polymer melt in circular tubes of varying dimensions and curved sections to determine how shape affects residual wall thickness uniformity. Huzyak and Koelling [6] and Gauri and Koelling [4] studied gas penetration through viscoelastic fluids. They used viscoelastic Boger fluids with constant Newtonian viscosity to test the effect of elasticity on coating film thickness. They noted that fluid elasticity affected the thickness of the coating film. Gauri and Koelling [4] also performed a PIV measurement to elucidate the velocity field near gas-bubble tips. Kamişli and Ryan [7] proposed a simple model based on a power-law viscosity function to analyze the gas-liquid dynamics of liquid displacement in circular tubes and noncircular channels. 
TABLE 1: Parameters of the Carreau-Yasuda viscosity model for the studied solutions.

\begin{tabular}{lcccccccc}
\hline Solution & $T\left({ }^{\circ} \mathrm{C}\right)$ & $\eta_{0}(\mathrm{~Pa} \cdot \mathrm{s})$ & $\eta_{\infty}(\mathrm{Pa} \cdot \mathrm{s})$ & $\lambda(\mathrm{s})$ & $a_{1}$ & $a_{2}$ & $\dot{\gamma}\left(\mathrm{s}^{-1}\right)$ & Average error $(\%)$ \\
\hline $1.5 \%$ CMC & 25 & 2.895 & 0.0719 & 0.2999 & 0.8695 & 0.4199 & $0.1-3000$ & 3.50 \\
$0.5 \%$ CMC & 25 & 0.232 & 0.0183 & 0.0002 & 0.1908 & 0.1404 & $0.1-3000$ & 0.79 \\
$1.5 \%$ PAA & 25 & 31.530 & 0.0476 & 7.709 & 75.651 & 0.2149 & $0.1-1000$ & 1.49 \\
$0.5 \%$ PAA & 25 & 3.902 & 0.0153 & 6.5964 & 8.2142 & 0.3479 & $0.1-1000$ & 1.52 \\
$0.5 \%$ PAS & 25 & 19.410 & 0.0532 & 5.9270 & 7.5677 & 0.2550 & $0.1-1000$ & 5.46 \\
$0.5 \%$ PAS & 25 & 2.150 & 0.0249 & 4.7794 & 5.1828 & 0.1332 & $0.1-1000$ & 1.03 \\
\hline
\end{tabular}

Bonn and Meunier [17] and Lindner et al. [8-10] described the effect of both shear-thinning viscosity and normal stress on finger growth in a Hele-Shaw cell. Yamamoto et al. [18] experimentally studied the effect of capillary number $(\mathrm{Ca})$ and Weissenberg number (Wi) on $m$ for Newtonian and nonNewtonian fluids.

The present study analyzed gas penetration through viscoelastic fluids with shear-thinning viscosity to elucidate the effect of shear-thinning properties on the gas-fluid interface. The tube contains sudden contraction and sudden expansion of two parts. An MFC is used to control the rate and volume of gas injection. Coating film thickness on tube walls is characterized by both $\mathrm{Ca}$ and $\mathrm{Wi}$, and these nondimensional groups are evaluated in terms of shear-thinning effect.

\section{Experiment}

2.1. Experimental Setup. Gas penetration in a glass tube was observed. Figure 1 shows the experimental setup for the gas penetration. It is modified from one adopted elsewhere [14]. The images of the bubble are captured by a high-speed camera (CASIO EX-F1) with 6 megapixel/60 frames per second. The light source is a coaxial light source (LA180-Me). The pressure at the entrance is $10 \mathrm{~kg} / \mathrm{cm}^{2}$. Nitrogen with $99.99 \%$ purity is selected as the injection gas. The volume rate of injection gas was controlled using MFC. The gas was injected at two volume rates $600 \mathrm{~mL} / \mathrm{min}$ and $200 \mathrm{~mL} / \mathrm{min}$. The tube, which is made of high-hardness heat-resistant glass, is shown in Figure 2. The dimensions of the tube are $L=1000 \mathrm{~mm}$, $L_{1}=200 \mathrm{~mm}, L_{2}=400 \mathrm{~mm}, d_{2}=8 \mathrm{~mm}$, and $d_{1}=$ $4 \mathrm{~mm}$. The inclined angle $\theta$ is $15^{\circ}, 45^{\circ}, 75^{\circ}$, and $90^{\circ}$, and $S$ represent measuring position, respectively. The tube was covered by a viewing box dimensions $50 \mathrm{~mm} \times 50 \mathrm{~mm} \times$ $1000 \mathrm{~mm}$. Glycerin has similar refraction index to that of the glass. A ruler was fixed to the frame for convenience of calculation of the velocity of the bubble.

2.2. Test Fluids. This study uses viscoelastic fluid. The viscoelastic fluids are $1.5 \mathrm{wt} \%$ and $0.5 \mathrm{wt} \%$ (polyacrylamide (PAA), carboxymethyl (CMC), and polyacrylsodium (PAS)). Rheological measurements were carried out with a cone-plate type rheometer (Gemini HR Nano). The cone is 2 degrees and diameter is $5.5 \mathrm{~mm}$. The measurements of rheological properties were carried out at $25^{\circ} \mathrm{C}$, which is the same temperature condition for the gas penetration experiments. All the viscoelastic fluids have shear-thinning viscosity and nonzero first normal stress difference. The shear viscosity of every viscoelastic fluid shows strong shear-thinning property.

The shear viscosity of the solutions, $\mu$, as a function of shear rate, $\dot{\gamma}$, is represented in Figure 3. Their data can be found in Table 1 . The Carreau-Yasuda viscosity model:

$$
\mu=\mu_{\infty}+\left(\mu_{0}-\mu_{\infty}\right)\left(1+(\lambda \dot{\gamma})^{a}\right)^{(n-1) / a},
$$

where $\mu$ is the measured viscosity, $\dot{\gamma}$ is the applied shear rate, $\mu_{0}$ is the viscosity limit when $\dot{\gamma} \rightarrow 0$, and $\mu_{\infty}$ is the viscosity at infinite shear rate, which was set as the solvent viscosity. The parameter $\lambda$ has units of time, and $n$ and a are dimensionless parameters described in Sousa et al. [13]. Figure 4 shows the shear rate and the first normal stress difference of the test fluids. Table 2 summarizes both surface tensions and the density $\rho$ of the test fluids at $25^{\circ} \mathrm{C}$. The surface tension was measured with Du Nouy (DST30) and the density $\rho$ was measured with (DA-130N).

We need to substitute $\dot{\gamma}=U / H$ to (1) to evaluate $\mu$. In the present analysis, we applied the average velocity of the bubble as $U$ and two definitions of $H$, (a) $H=d_{1}$ and (b) $H=d_{2}$ to estimate the shear rate.

2.3. Experimental Method. Ha-Ngoc and Fabre [5] simulated the shape of the bubbles with dimensionless parameters, such as length and height numerically. Sousa et al. [13] captured image of the bubble penetrating through the non-Newtonian fluid polyacrylamide (PAA) in a vertical tube by a CCD camera experimentally. They also plot the bubble profiles in some dimensionless parameters. In this experiment, the gas is injected into tube filled with various viscoelastic fluids in an isothermal environment. The volume rate of the injection gas was controlled by MFC in this experiment. The profile of the bubble is captured by a high-speed camera. The fractional ratio $(m)$ of the liquid was estimated by these photographs. Figure 5 shows a photo before the image processing and the ultimate graphics generated by the image processing. The width of the bubble was estimated by these images.

The photographs captured by the camera are processed by the image processing program written in the Matlab to measure the dimensions of the bubble accurately. Some binarization and skeletonization skills are applied to eliminate the errors caused by light shadow. The bubble profiles in some dimensionless parameters are plotted. The bubble profile information is obtained by using the Level Set Method. In this study, we study the impact of the various flow rates and various non-Newton fluids on the bubble velocity $(U)$ and the dimensionless parameters, such as the $\mathrm{Ca}, \mathrm{Wi}$, and $m$. 


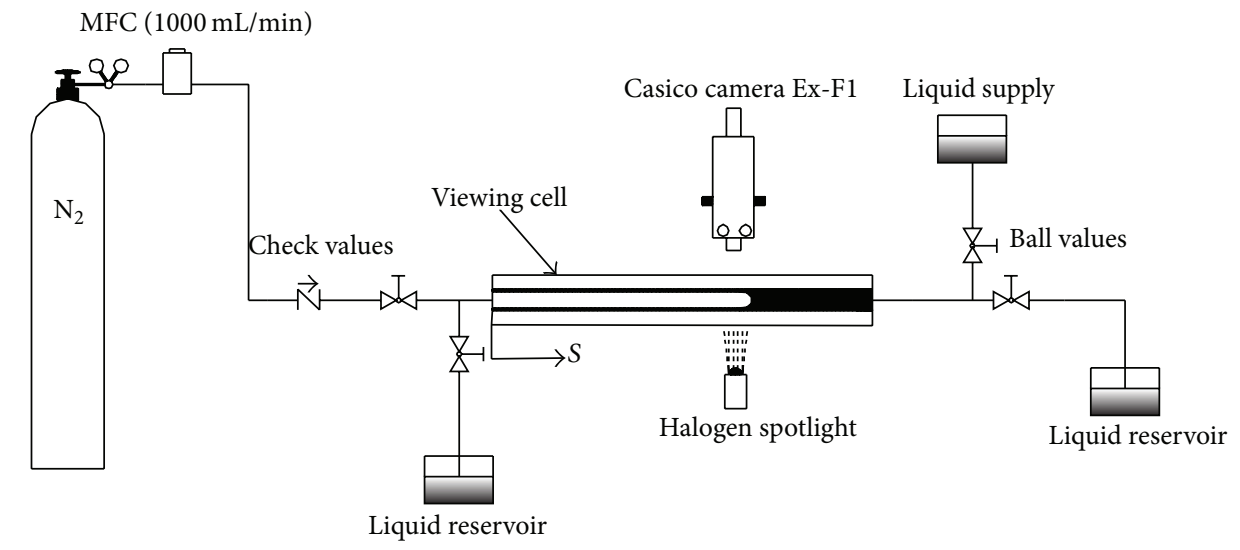

Figure 1: Experimental system.

TABLE 2: Physical properties of viscoelastic fluid.

\begin{tabular}{lccc}
\hline Solution & $T\left({ }^{\circ} \mathrm{C}\right)$ & Density $\rho\left(\mathrm{kg} / \mathrm{cm}^{3}\right)$ & Surface tension $\sigma(\mathrm{mN} / \mathrm{m})$ \\
\hline $1.5 \mathrm{wt} \%$ CMC in water & 25 & 1004.07 & 73.7 \\
$0.5 \mathrm{wt} \%$ CMC in water & 25 & 945.07 & 73.3 \\
$1.5 \mathrm{wt} \%$ PAA in water & 25 & 1008.87 & 73.0 \\
$0.5 \mathrm{wt} \%$ PAA in water & 25 & 989.03 & 71.0 \\
$1.5 \mathrm{wt} \%$ PAS in water & 25 & 998.67 & 74.4 \\
$0.5 \mathrm{wt} \%$ PAS in water & 25 & 991.83 & 69.1 \\
\hline
\end{tabular}

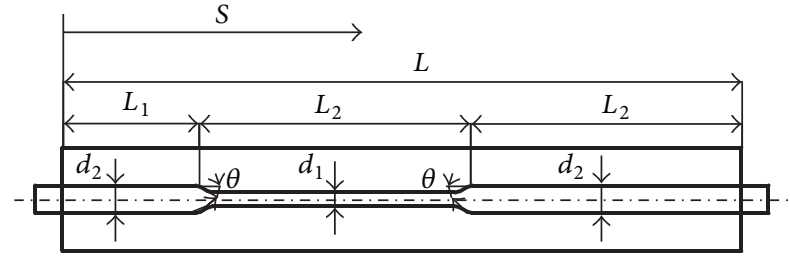

FIGURE 2: Schematic of the experimental tube.

The dimensionless parameters are defined as

$$
\begin{aligned}
\mathrm{Ca} & =\frac{\eta(\dot{\gamma}) U}{\sigma}, \\
m & =\frac{R_{o}^{2}-R_{b}^{2}}{R_{o}^{2}}, \\
R & =\frac{N_{1}}{2 \eta(\dot{\gamma}) \dot{\gamma}^{2}}, \\
\mathrm{Wi} & =\frac{R U}{H},
\end{aligned}
$$

where $U$ is the average velocity of the bubble, $\mathrm{Ca}$ is capillary number, $\eta(\dot{\gamma})$ is the shear viscosity of the viscoelastic fluids, $\sigma$ is the surface tension of the viscoelastic fluids, $\dot{\gamma}$ is shear rate, $m$ is the fractional ratio, $R_{b}$ is the radius of the bubble, $R_{o}$ is the internal radius of the circular tube, $R$ is the Maxwell type relaxation time, $N_{1}$ is the first normal stress difference, and $H$ is representative length.

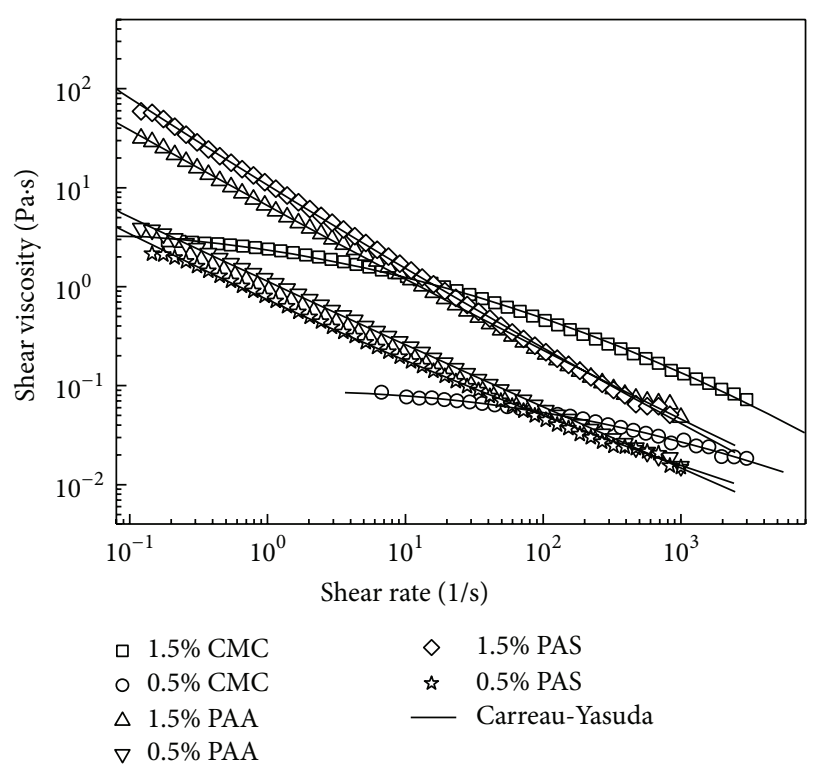

FIGURE 3: The shear viscosity as a function of the shear rate for the studied solutions and Carreau-Yasuda model fitted curves.

We need to determine a representative shear rate $\dot{\gamma}=$ $U / H$ to evaluate $\eta(\dot{\gamma})$ and $R$. In the present analysis, we applied the average velocity of the bubble as $U$ and two definitions of $H$, (a) $H=d_{1}$ and (b) $H=d_{2}$ to estimate the shear rate. 


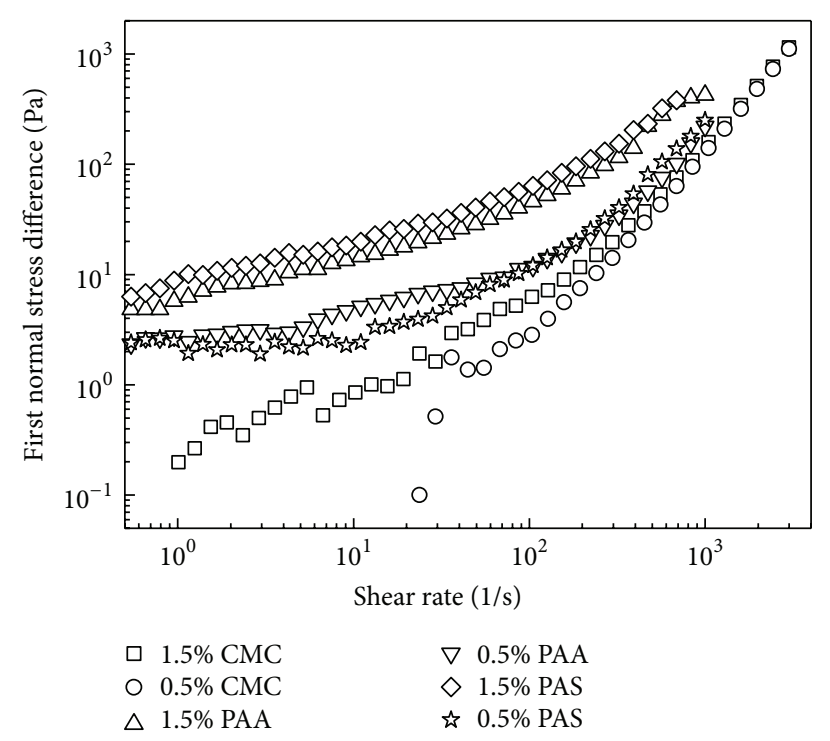

FIGURE 4: The first normal stress difference as a function of the shear rate for the studied solutions.

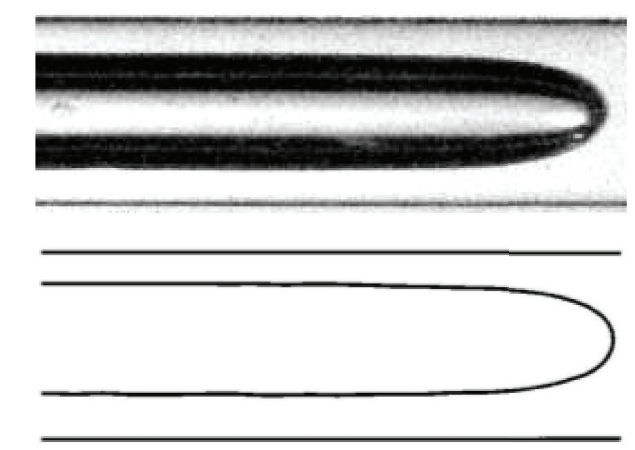

FIGURE 5: Bubble image before and after processing.

\section{Results and Discussion}

3.1. Bubble Velocity. The test tube is shown in Figure 2. The entrance section, sudden contraction section, and sudden expansion section are in the sections from 0 to $20 \mathrm{~cm}$, from 20 to $60 \mathrm{~cm}$, and from 60 to $100 \mathrm{~cm}$, respectively. In this study, two different nitrogen flow rates $(600 \mathrm{~mL} / \mathrm{min}, 200 \mathrm{~mL} / \mathrm{min})$ were controlled by MFC. Inlet pressure was $10 \mathrm{~kg} / \mathrm{cm}^{2}$, the diameter of sudden expansion section and entrance section was $8 \mathrm{~mm}$, and the diameter of the sudden contraction section was $4 \mathrm{~mm}$. Figures 6 and 7 show that bubble velocity increased as sudden contraction tube angle increased. When flow rate was fixed, bubble velocity increased as contraction angle increased.

The bubble moved fastest at $S=60 \mathrm{~cm}$ in the contraction section. Bubble velocity for $1.5 \mathrm{wt} \% \mathrm{CMC}$ was higher than that for $0.5 \mathrm{wt} \% \mathrm{CMC}$. As contraction angle increased, bubble velocity for $1.5 \mathrm{wt} \% \mathrm{CMC}$ was significantly higher than that for $0.5 \mathrm{wt} \% \mathrm{CMC}$. The main cause of this phenomenon is fixed MFC flow and $10 \mathrm{~kg} / \mathrm{cm}^{2}$ inlet pressure. Under the given constant flow rate, the low shear viscosity fluid could be expelled more easily due to reduced resistance. To

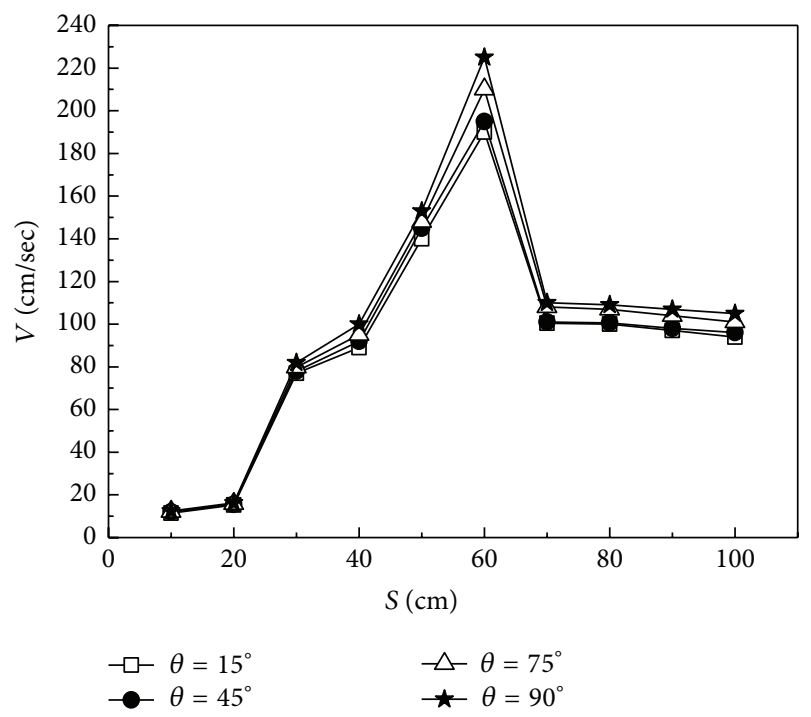

Figure 6: Position of bubble front corresponds to the velocity of bubble for $1.5 \mathrm{wt} \% \mathrm{CMC}$ with gas flow $600 \mathrm{~mL} / \mathrm{min}$.

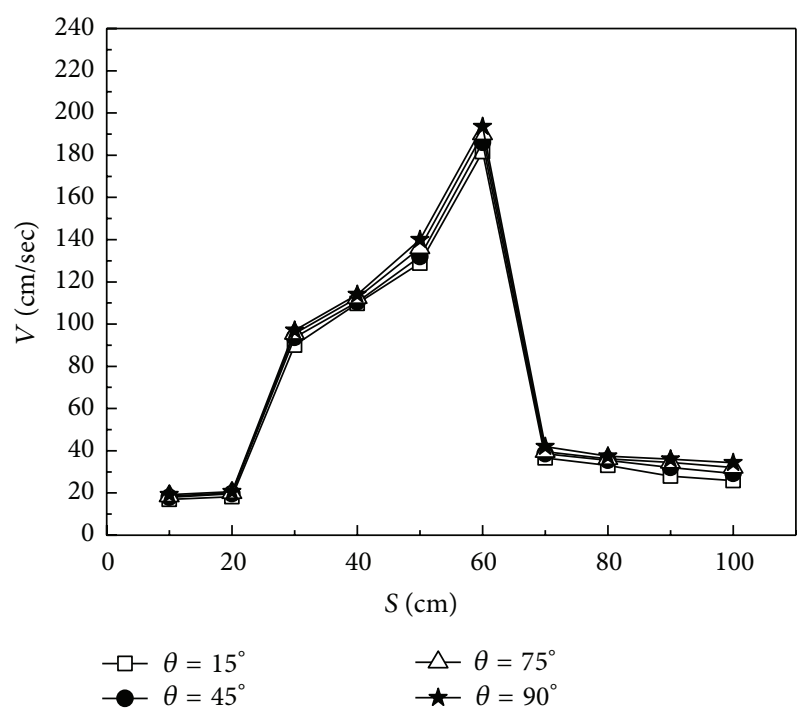

FIgURE 7: Position of bubble front corresponds to the velocity of bubble for $0.5 \mathrm{wt} \% \mathrm{CMC}$ with gas flow $600 \mathrm{~mL} / \mathrm{min}$.

promote the viscoelastic fluid move, injection gas pressure was reduced below $10 \mathrm{~kg} / \mathrm{cm}^{2}$. According to the law of inertia, the pressure becomes smaller, following the smaller force. So relatively reducing the acceleration, the velocity is steady. However, since gas flow remained constant and the increased resistance caused by increased viscoelastic fluid shear viscosity caused fluid pressure to increase to $10 \mathrm{~kg} / \mathrm{cm}^{2}$, bubble velocity increased for the higher force introduced by the higher pressure, which is consistent with the law of inertia.

Figure 8 shows that the velocity of bubble was in the sequence of PAS > PAA > CMC for $1.5 \mathrm{wt} \%$ viscoelastic fluids from entrance to sudden contraction tube. Here, the shear rate was lower than $20 / \mathrm{s}$. When the bubble entered the 


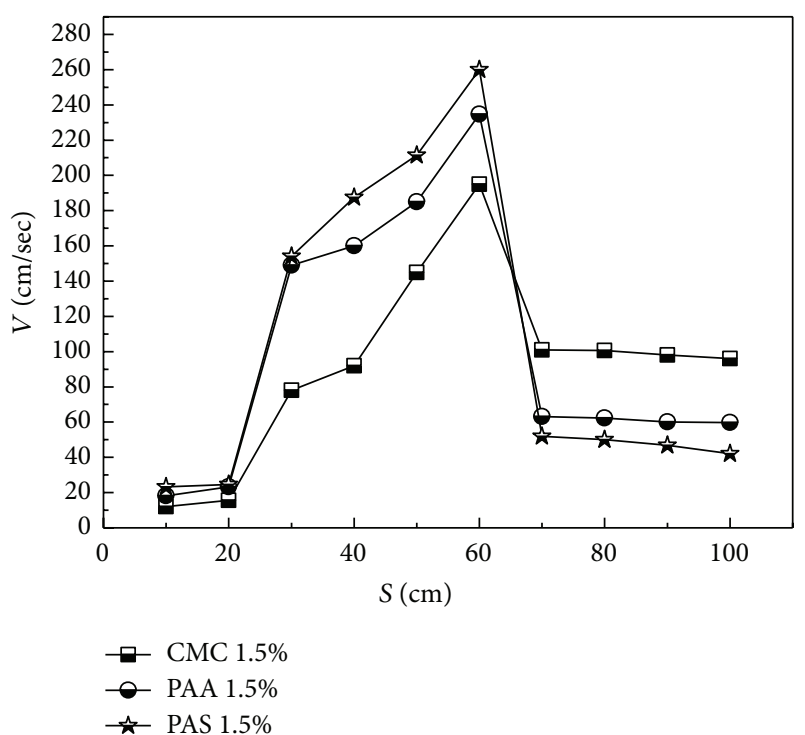

FIGURE 8: Position of bubble front corresponds to the velocity of bubble for $1.5 \mathrm{wt} \%$ viscoelastic fluids $\left(Q=600 \mathrm{~mL} / \mathrm{min}\right.$ and $\left.\theta=45^{\circ}\right)$.

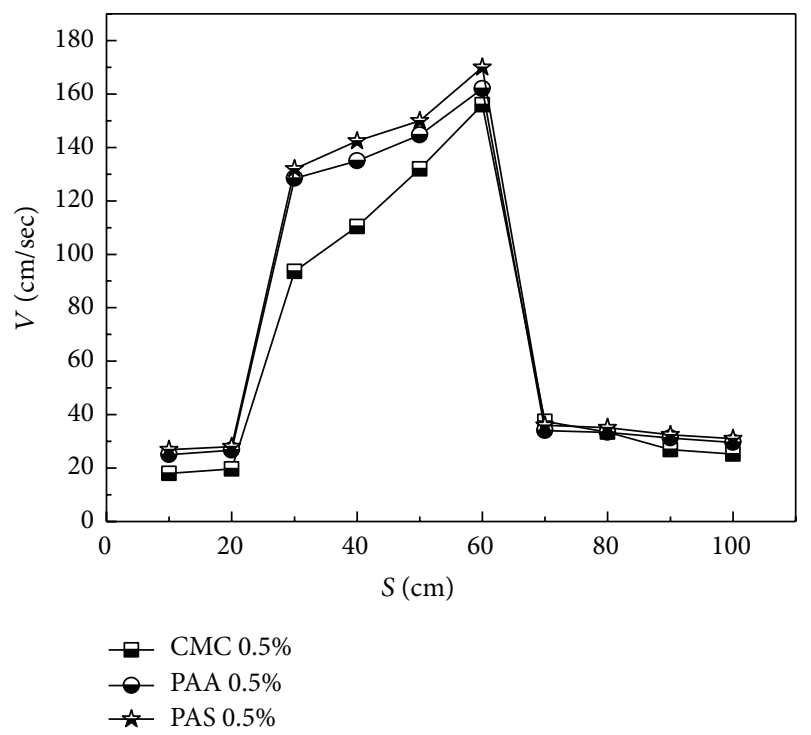

FIgUre 9: Position of bubble front corresponds to the velocity of bubble for $0.5 \mathrm{wt} \%$ viscoelastic fluids $\left(Q=600 \mathrm{~mL} / \mathrm{min}\right.$ and $\left.\theta=45^{\circ}\right)$.

sudden expansion section, its velocity was in the sequence of $\mathrm{CMC}>\mathrm{PAA}>\mathrm{PAS}$. Here, the shear rate exceeded 20/s. The difference was caused by shear viscosity. Figure 3 shows the main cause of the phenomenon. When viscoelastic fluid was $1.5 \mathrm{wt} \%$ and shear rate was lower than $20 / \mathrm{s}$, the shear viscosity was in the sequence of PAS > PAA > CMC. However, the shear rate exceeded 20/s, and the shear viscosity was in the sequence CMC > PAA > PAS. Figure 9 shows that bubble velocity was in the sequence PAS > PAA > CMC for $0.5 \mathrm{wt} \%$ viscoelastic fluids from entrance to sudden contraction section. When bubbles stretched to the sudden

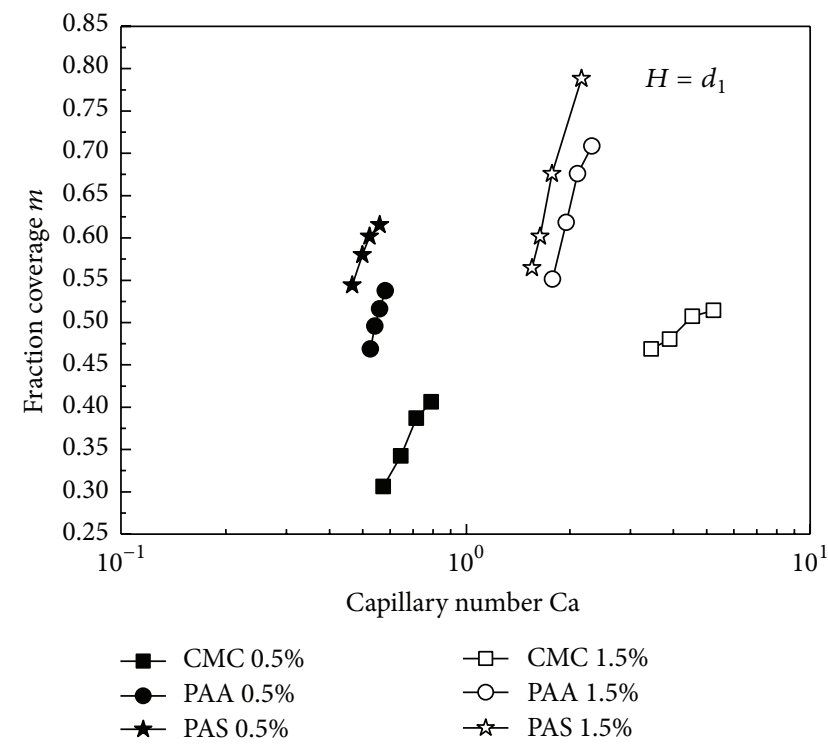

FIGURE 10: Fractional coverage $m$ as a function of capillary number Ca when sudden contraction tube angle is $\theta=45^{\circ}, Q=600 \mathrm{~mL} / \mathrm{min}$ and $H=d_{1}$.

expansion section, bubble velocities were similar. Therefore, the shear-thinning behaviors of viscoelastic fluids have an important role in bubble velocity.

3.2. Relationship between $\mathrm{Ca}$ and $m$. Figure 10 shows the relationship of $m$ and $\mathrm{Ca}$ in sudden contraction tube with $H$ was case (a). The result shows that $\mathrm{Ca}$ value increases and $m$ also increases. Here, the highest rate of increase in $m$ was $1.5 \mathrm{wt} \%$ PAS. For $1.5 \mathrm{wt} \%$ viscoelastic fluid, all Ca values exceeded 1. The maximum value of Ca was 5.24 for $1.5 \mathrm{wt} \%$ CMC at $S=60 \mathrm{~cm}$, and the maximum value of $m$ was 0.79 for $1.5 \mathrm{wt} \% \mathrm{PAS}$ at $S=60 \mathrm{~cm}$. For $0.5 \mathrm{wt} \%$ viscoelastic fluid, all $\mathrm{Ca}$ values were less than 1 . The minimum value of $\mathrm{Ca}$ was 0.46 for $0.5 \mathrm{wt} \%$ PAS at $S=30 \mathrm{~cm}$, and the minimum value of $m$ was 0.31 for $0.5 \mathrm{wt} \% \mathrm{CMC}$ at $S=30 \mathrm{~cm}$. These experimental results agree with those reported in previous studies [14] of viscoelastic fluids. Figure 2 shows the main causes of these phenomena. For $1.5 \mathrm{wt} \%$ viscoelastic fluids with shear rates higher than 20/s and $0.5 \mathrm{wt} \%$ viscoelastic fluids with shear rates higher than 100/s, the shear viscosity sequence was CMC $>$ PAA > PAS. Figures 8 and 9 show the velocity of bubble increases for sudden contraction tube. The shear rate was calculated by $\dot{\gamma}=U / H . U$ and $\eta(\dot{\gamma})$ were measured to obtain the value of $\mathrm{Ca}$ according to (1). Because the values of $U$ and $\eta(\dot{\gamma})$ were larger, $\mathrm{Ca}$ for $\mathrm{CMC}$ was also larger. Figure 3 shows that the value of $m$ was related to shear viscosity. Figure 10 shows that as the shear viscosity increased bubble diameter correlated negatively with $m$. The value of $m$ was related to the shear viscosity.

Figure 11 shows the relationship of $m$ and $\mathrm{Ca}$ in sudden expansion tube with $H$ was case (b). Because the definition of the representative shear rate differed from that in case (a), the plot shift was in the direction of the Ca axis. However, qualitative analysis obtained the same results as in case (a). 


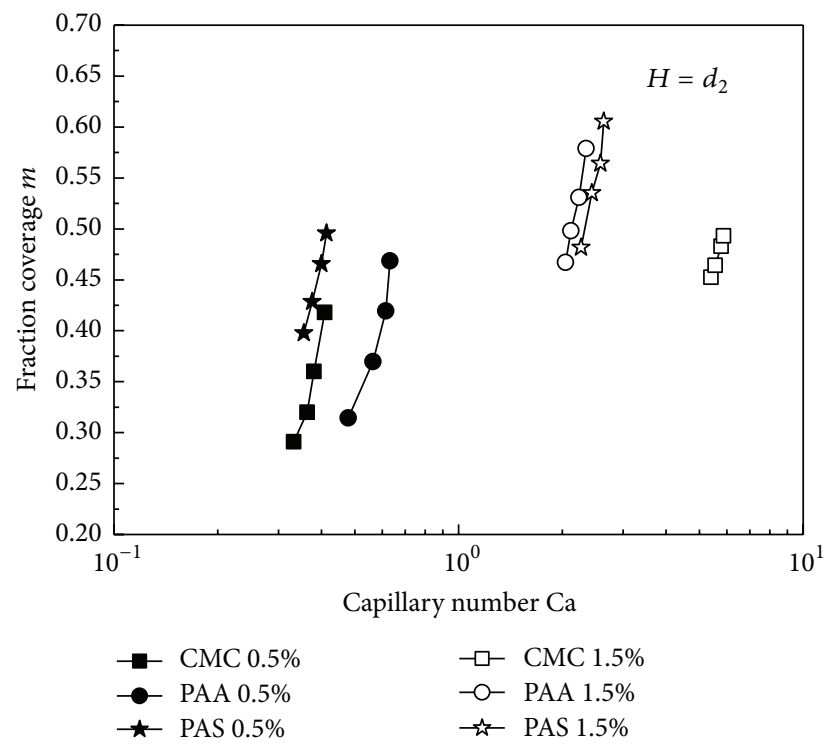

FIGURE 11: Fractional coverage $m$ as a function of capillary number Ca when sudden expansion tube angle is $\theta=45^{\circ}, Q=600 \mathrm{~mL} / \mathrm{min}$ and $H=d_{2}$.

The experimental results showed the reported relationship between $m$ and $\mathrm{Ca}$ for fluids with shear-thinning viscosity when evaluating $\mathrm{Ca}$ as a function of shear rate. For $1.5 \mathrm{wt} \%$ viscoelastic fluid, all $\mathrm{Ca}$ values exceeded 1 . The maximum Ca value was 5.88 for $1.5 \mathrm{wt} \% \mathrm{CMC}$ at $S=60 \mathrm{~cm}$, and the maximum $m$ value was 0.61 for $1.5 \mathrm{wt} \%$ PAS at $S=60 \mathrm{~cm}$. For $0.5 \mathrm{wt} \%$ viscoelastic fluid, all Ca values were lower than 1. The minimum value of $\mathrm{Ca}$ was 0.33 for $0.5 \mathrm{wt} \% \mathrm{CMC}$ at $S=30 \mathrm{~cm}$, and the minimum value of $m$ was 0.29 for $0.5 \mathrm{wt} \%$ $\mathrm{CMC}$ at $S=30 \mathrm{~cm}$. The value of $\mathrm{Ca}$ changed for $0.5 \mathrm{wt} \%$ viscoelastic fluids as described in Figure 10. Figure 3 shows that, for viscoelastic fluids with $0.5 \mathrm{wt} \%$ and shear rate lower than $100 /$ s, the shear viscosity was in the sequence PAA > PAS $>$ CMC. Figures 8 and 9 show that bubble velocity was lower for the sudden expansion tube. To evaluate the value of shear rate as being $\dot{\gamma}=U / H$, it was less than $100 /$ s. $U$ and $\eta(\dot{\gamma})$ were measured to obtain the Ca according to (1). Because the values of $U$ and $\eta(\dot{\gamma})$ were larger, Ca was also larger for PAA. $U$ and $\eta(\dot{\gamma})$ values significantly affected $\mathrm{Ca}$, and shear viscosity significantly affected $m$.

3.3. Relationship between Wi and $m / m_{N}$. In accordance with the discussion of Deborah number and $m / m_{N}$ correlation in Huzyak and Koelling [6], the current study defined Deborah number approaching 5 as fractional ratio 30\% more than that in the Newtonian case. Yamamoto et al. [18] studied Weissenberg number and $m / m_{N}$ correlation. They demonstrated that the film becomes thinner than that in the Newtonian case when Wi is small and the viscous effect is dominant. $m_{N}$ was defined as the fractional ratio of Newtonian fluid at the corresponding Ca. Figures 12 and 13 show the relationship between fractional ratio $m / m_{N}$ and Wi in cases (a) and (b), respectively. Here, $m_{N}$ was the fractional ratio of viscoelastic fluids at $S=40 \mathrm{~cm}$ and at $S=80 \mathrm{~cm}$ for the sudden

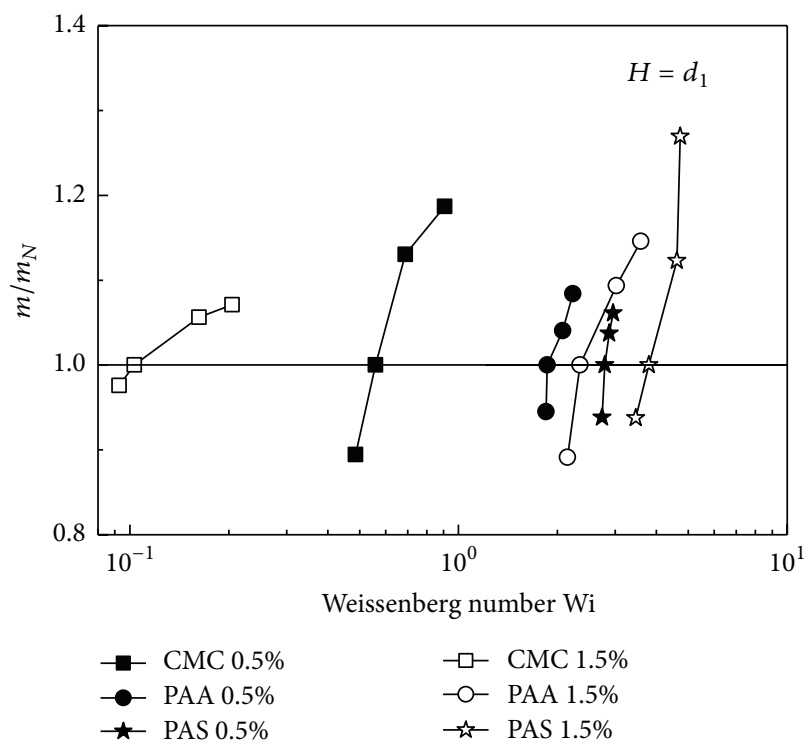

FIGURE 12: Increase of fractional coverage $\mathrm{m} / \mathrm{m}_{N}$ as a function of Weissenberg number Wi when sudden contraction tube angle is $\theta=$ $45^{\circ}, \mathrm{Q}=600 \mathrm{~mL} / \mathrm{min}$ and $H=d_{1}$.

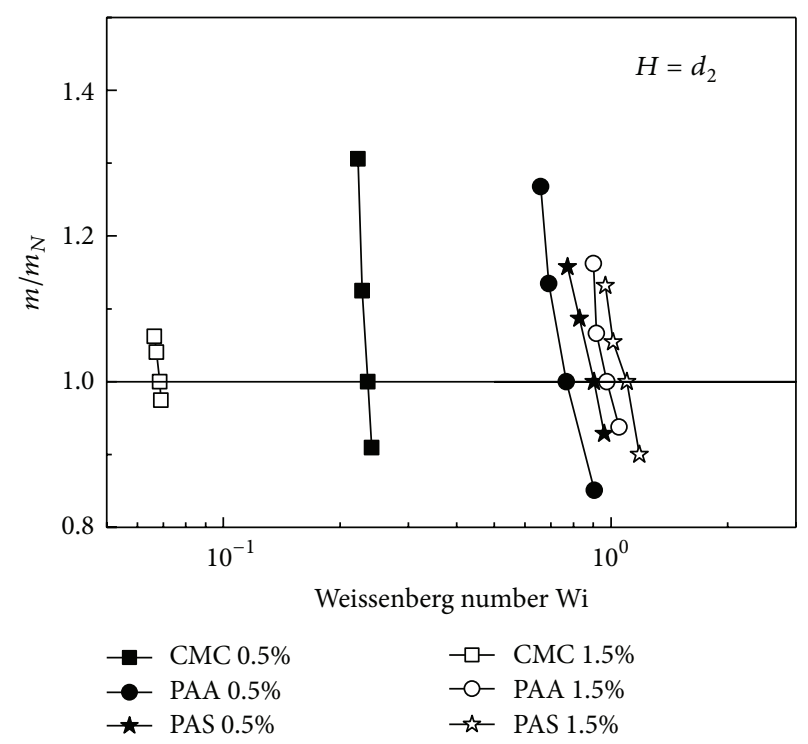

FIGURE 13: Increase of fractional coverage $\mathrm{m} / \mathrm{m}_{N}$ as a function of Weissenberg number Wi when sudden contraction tube angle is $\theta=$ $45^{\circ}, \mathrm{Q}=600 \mathrm{~mL} / \mathrm{min}$ and $H=d_{2}$.

contraction tube and the sudden expansion tube, respectively. The condition $m / m_{N}>1$ means the coating film is increased, and $m / m_{N}<1$ means the coating film is decreased when the bubble moves.

Figure 12 shows bubble movement from $S=40 \mathrm{~cm}$ to $S=$ $60 \mathrm{~cm}$. The increase in $\mathrm{m} / \mathrm{m}_{N}$ for $1.5 \mathrm{wt} \%$ viscoelastic fluids exhibited the following relationship: PAS > PAA > CMC. When the bubble moved to $S=60 \mathrm{~cm}$, the maximum ratio of $m / m_{N}$ was 1.27 for PAS. The minimum ratio of $m / m_{N}$ was 1.07 for CMC. The rate of increase in $m / m_{N}$ for $1.5 \mathrm{wt} \%$ viscoelastic fluids exhibited the following relationship: CMC 
TABLE 3: Evolution of bubble contours for sudden contraction angle $\theta=15^{\circ}$.

Solution Sudden contraction angle $\left(\theta=15^{\circ}\right)$

TABLE 4: Evolution of bubble contours for sudden contraction angle $\theta=90^{\circ}$.

Solution Sudden contraction angle $\left(\theta=90^{\circ}\right)$

$>$ PAA > PAS. When the bubble moved to $S=60 \mathrm{~cm}$, the maximum ratio of $\mathrm{m} / \mathrm{m}_{N}$ was 1.18 for CMC. The minimum ratio of $m / m_{N}$ was 1.06 for PAS. Figure 13 shows the bubble movement from $S=80 \mathrm{~cm}$ to $S=100 \mathrm{~cm}$. The rate of increase in $m / m_{N}$ of $1.5 \mathrm{wt} \%$ viscoelastic fluids exhibited the following relationship: PAA > PAS > CMC. When the bubble moved to $S=100 \mathrm{~cm}$, the maximum ratio of $m / m_{N}$ was 1.16 for PAA. The minimum ratio of $m / m_{N}$ was 1.06 for CMC. The rate of increases in $m / m_{N}$ of $0.5 \mathrm{wt} \%$ viscoelastic fluids exhibited the following relationship: $\mathrm{CMC}>\mathrm{PAA}>\mathrm{PAS}$. When the bubble moved to $S=100 \mathrm{~cm}$, the maximum ratio of $m / m_{N}$ was 1.31 for CMC. The minimum ratio of $m / m_{N}$ was 1.15 for CMC.

These different phenomena shown by Figures 12 and 13 were resulting from shear viscosity. When the shear viscosity of viscoelastic fluids is higher, bubble movement increased, and $m / m_{N}$ increases rate is larger. The shear viscosity value had an important effect on $\mathrm{m} / \mathrm{m}_{N}$. When wt\% was equal to that in Figure 4, the value of normal force was in the sequence PAS $>$ PAA $>$ CMC. Figures 12 and 13 show that the value of Wi was in the sequence PAS > PAA > CMC of the same wt $\%$. The effect of elastic normal force can be explained as follows [12]. Normal force exists in the radial direction. The direction of force is towards the centerline, and the force becomes larger with increasing the shear rate or the velocity gradient. The value of normal force therefore has an important role in $\mathrm{Wi}$.
3.4. Bubble Profiles. An early study by Saffman and Taylor [11] captured the bubble profile using a camera as the bubble penetrated a Hele-Shaw cell and presented a parametric equation. $\mathrm{Bi}$ and Zhao [12] used a camera to record the evolution of bubbles in three tubes. Figure 3 shows that the viscoelastic fluids were $0.5 \mathrm{wt} \%$, and shear rate was lower than $100 /$ s. Shear viscosity was in the sequence of PAA $>$ PAS > CMC. Tables 3 and 4 show that gas flow was $200 \mathrm{~mL} / \mathrm{min}$ and shear rate was lower than $100 / \mathrm{s}$. When the shear viscosity increased, the bubble front is sharper in the sudden contraction tube. When gas flow was $600 \mathrm{~mL} / \mathrm{min}$, as the shear viscosity increased the bubble front shape is close to torch shape in the sudden contraction tube. As for the effects of MFC, bubble velocity increased when viscoelastic fluids viscosity increased.

Tables 5 and 6 show the continuous variation in bubble shape observed in the sudden expansion tube. As shear viscosity increased, gas flow increased to $200 \mathrm{~mL} / \mathrm{min}$, and the bubble front became less convex. As shear viscosity increased, gas flow increased to $600 \mathrm{~mL} / \mathrm{min}$, and the bubble front is close to torch shape. The photographs in Figures 14 and 15 were referenced in [18] and processed by the image processing program in Matlab. The bubble profiles, which were obtained by Level Set Method, show that bubble shape correlated with the condition of viscoelastic fluids at a certain 
TABLE 5: Evolution of bubble contours for sudden expansion angle $\theta=15^{\circ}$.

Solution Sudden expansion angle $\left(\theta=15^{\circ}\right)$

TABLE 6: Evolution of bubble contours for sudden expansion angle $\theta=90^{\circ}$.

Solution

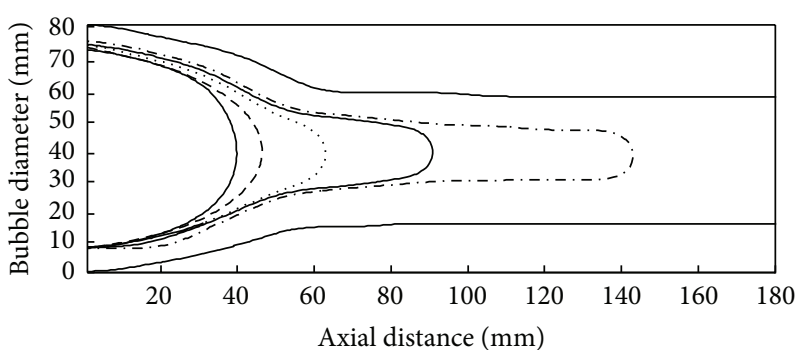

FIGURE 14: Bubble shape continuous variation in $0.5 \mathrm{wt} \%$ PAA for sudden contraction angle $\theta=15^{\circ}$ and $200 \mathrm{~mL} / \mathrm{min}$.

Q (200 $\mathrm{mL} / \mathrm{min})$ as gas was injected from the left side of the test tube. The horizontal axis represents the distance that the bubble moved, and the vertical axis represents the changes in bubble diameter. Figures 14 and 15 show that bubble velocity increased as wt $\%$ of PAA increased. The main reason is that the shear viscosity of viscoelastic fluids increases when gas flow is fixed.

\section{Conclusion}

This study analyzed gas penetration through viscoelastic fluids in a sudden contraction and sudden expansion tube. The experimental results indicated that viscoelastic properties

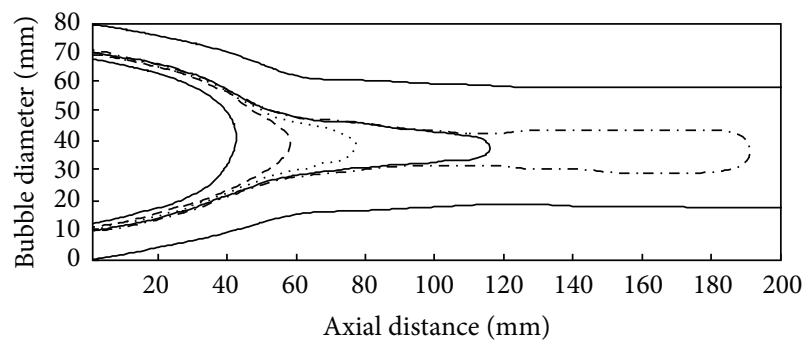

Figure 15: Bubble shape continuous variation in $1.5 \mathrm{wt} \%$ PAA for sudden contraction angle $\theta=15^{\circ}$ and $200 \mathrm{~mL} / \mathrm{min}$.

affect film thickness on the inner wall of the tube. In viscoelastic fluids, as shear viscosity is higher under a constant gas flow, the incline angle is bigger of tube, and bubble velocity increases. The analytical results showed that bubble velocity indicates the effect of shear-thinning viscosity and incline angle of tube. In viscoelastic fluids with $1.5 \mathrm{wt} \%, \theta=45^{\circ}$, and $Q=600(\mathrm{~mL} / \mathrm{min})$, the sudden contraction and sudden expansion in the range of $\mathrm{Ca}$ exceeded 1 . In viscoelastic fluids with $0.5 \mathrm{wt} \%, \theta=45^{\circ}$, and $Q=600(\mathrm{~mL} / \mathrm{min})$, the sudden contraction and sudden expansion in the range of $\mathrm{Ca}$ were less than 1 . The shear-thinning viscosity has an important effect on $\mathrm{Ca}$. Wi of CMC in a suddenly contracting or suddenly expanding tube is lower than other viscoelastic 
fluids; the main reason is that the normal force of the CMC is smaller. As the shear viscosity of the viscoelastic fluids increases, the rate of increase in $\mathrm{m} / \mathrm{m}_{N}$ also increases as the bubble moves. For a gas flow rate of $200 \mathrm{~mL} / \mathrm{min}$, as shear viscosity increases, the front of the bubble becomes more sharper in the contraction tube but blunter in the sudden expansion tube. For a flow rate of $600 \mathrm{~mL} / \mathrm{min}$, as shear viscosity increases, the front of the bubble becomes blunter in the contraction tube and exhibits a torch shape in the sudden expansion tube.

\section{Competing Interests}

The authors declare that they have no competing interests.

\section{References}

[1] F. P. Bretherton, “The motion of long bubbles in tubes," Journal of Fluid Mechanics, vol. 10, pp. 166-188, 1961.

[2] B. G. Cox, "On driving a viscous fluid out of a tube," Journal of Fluid Mechanics, vol. 14, no. 1, pp. 81-96, 1962.

[3] B. G. Cox, "An experimental investigation of the streamlines in viscous fluid expelled from a tube," Journal of Fluid Mechanics, vol. 20, article 193, 1964.

[4] V. Gauri and K. W. Koelling, "Gas-assisted displacement of viscoelastic fluids: flow dynamics at the bubble front," Journal of Non-Newtonian Fluid Mechanics, vol. 83, no. 3, pp. 183-203, 1999.

[5] H. Ha-Ngoc and J. Fabre, "A boundary element method for calculating the shape and velocity of two-dimensional long bubble in stagnant and flowing liquid," Engineering Analysis with Boundary Elements, vol. 30, no. 7, pp. 539-552, 2006.

[6] P. C. Huzyak and K. W. Koelling, "The penetration of a long bubble through a viscoelastic fluid in a tube," Journal of NonNewtonian Fluid Mechanics, vol. 71, no. 1-2, pp. 73-88, 1997.

[7] F. Kamişli and M. E. Ryan, "Gas-assisted non-newtonian fluid displacement in circular tubes and noncircular channels," Chemical Engineering Science, vol. 56, no. 16, pp. 4913-4928, 2001.

[8] A. Lindner, D. Bonn, and J. Meunier, "Viscous fingering in complex fluids," Journal of Physics: Condensed Matter, vol. 12, no. 8, pp. A477-A482, 2000.

[9] A. Lindner, D. Bonn, and J. Meunier, "Viscous fingering in a shear-thinning fluid," Physics of Fluids, vol. 12, no. 2, pp. 256261, 2000.

[10] A. Lindner, D. Bonn, E. C. Poire, M. B. Amar, and J. Meunier, "Viscous fingering in non-Newtonian fluids," Journal of Fluid Mechanics, vol. 469, no. 1, pp. 237-256, 2002.

[11] P. G. Saffman and G. Taylor, "The penetration of a fluid into a porous medium or Hele-Shaw cell containing a more viscous liquid," Proceedings of the Royal Society of London A, vol. 245, pp. 312-329, 1958.

[12] Q. C. Bi and T. S. Zhao, "Taylor bubbles in miniaturized circular and noncircular channels," International Journal of Multiphase Flow, vol. 27, no. 3, pp. 561-570, 2001.

[13] R. G. Sousa, M. L. Riethmuller, A. M. F. R. Pinto, and J. B. L. M. Campos, "Flow around individual Taylor bubbles rising in stagnant polyacrylamide (PAA) solutions," Journal of NonNewtonian Fluid Mechanics, vol. 135, no. 1, pp. 16-31, 2006.
[14] G. I. Taylor, "Deposition of a viscous fluid on the wall of a tube," Journal of Fluid Mechanics, vol. 10, no. 2, pp. 161-165, 1961.

[15] L. W. Schwartz, H. M. Princen, and A. D. Kiss, "On the motion of bubbles in capillary tubes," Journal of Fluid Mechanics, vol. 172, pp. 259-275, 1986.

[16] S. Y. Yang and H. L. Chou, "Study on the residual wall thickness at dimensional transitions and curved sections in gas-assisted molded circular tubes," Polymer Engineering and Science, vol. 42, no. 1, pp. 111-119, 2002.

[17] D. Bonn and J. Meunier, "Viscoelastic free-boundary problems: non-newtonian viscosity vs normal stress effects," Physical Review Letters, vol. 79, article 2662, 1997.

[18] T. Yamamoto, T. Suga, K. Nakamura, and N. Moti, “The gas penetration through viscoelastic fluids with shear-thinning viscosity in a tube," Journal of Fluids Engineering, vol. 126, no. 2, pp. 148-152, 2004. 

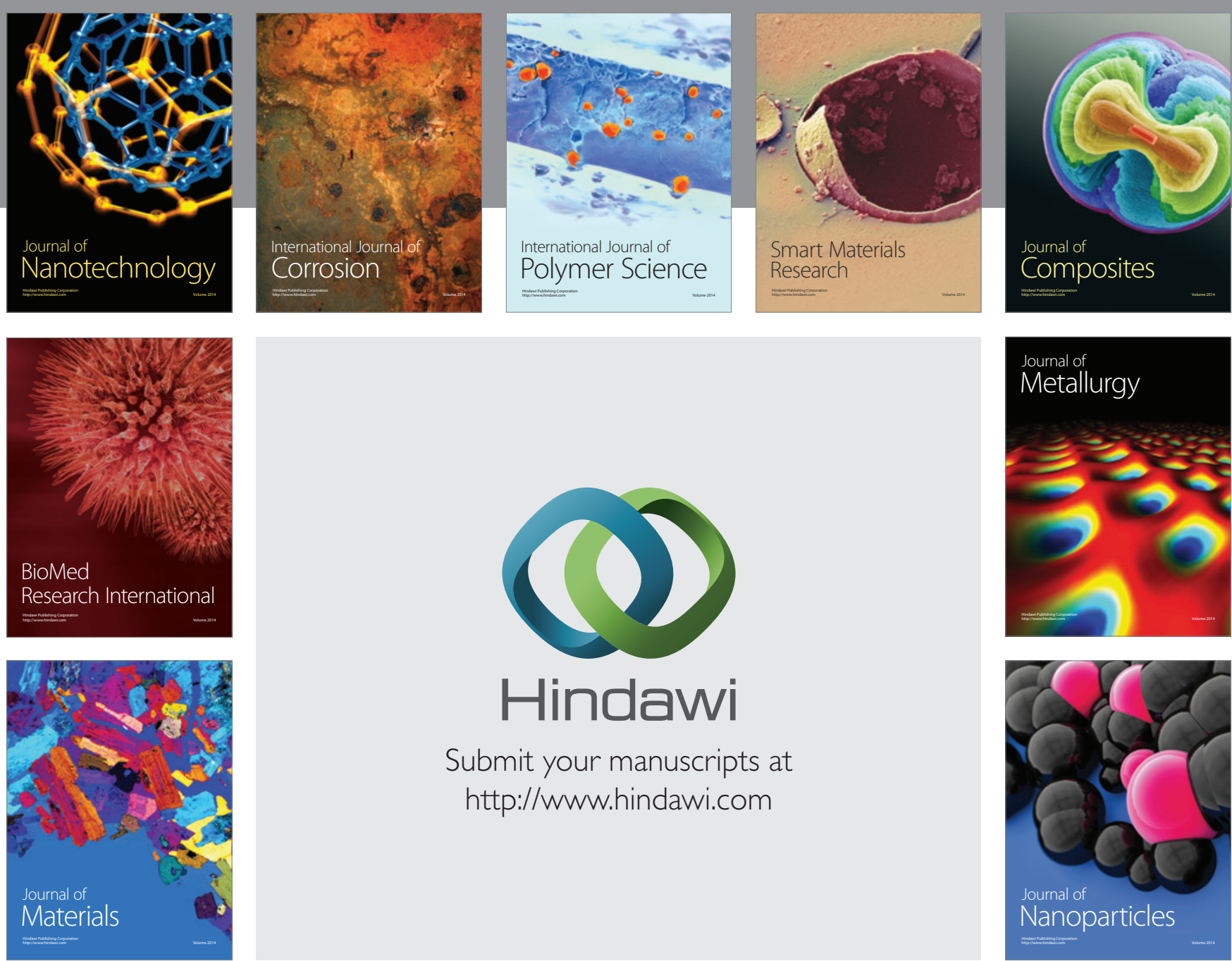

\section{Hindawi}

Submit your manuscripts at

http://www.hindawi.com

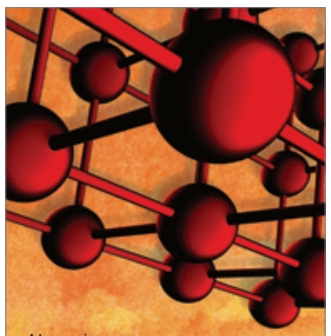

Materials Science and Engineering
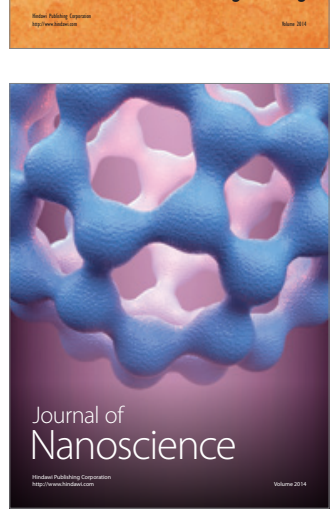
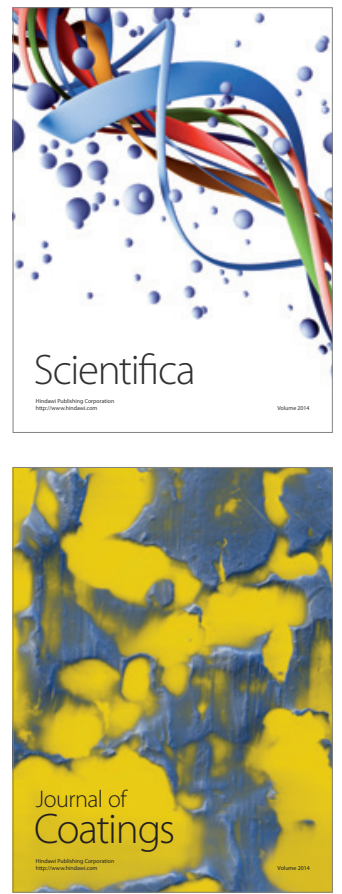
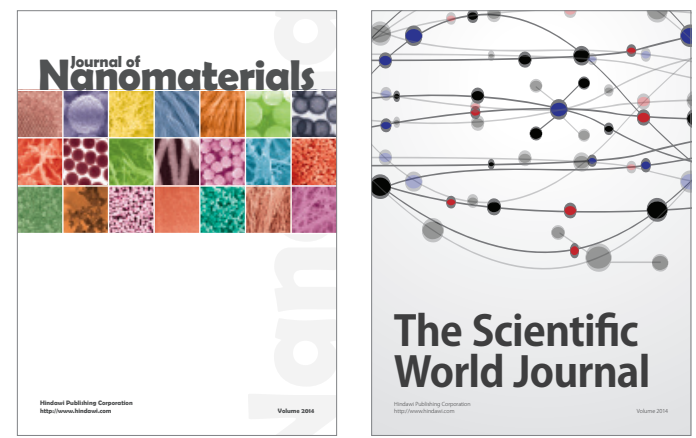

The Scientific World Journal
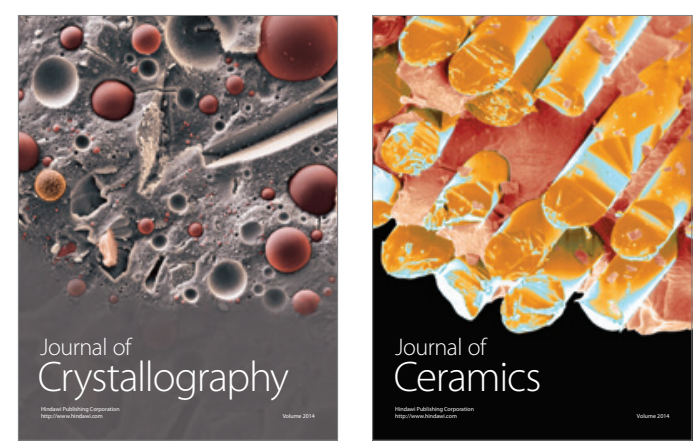
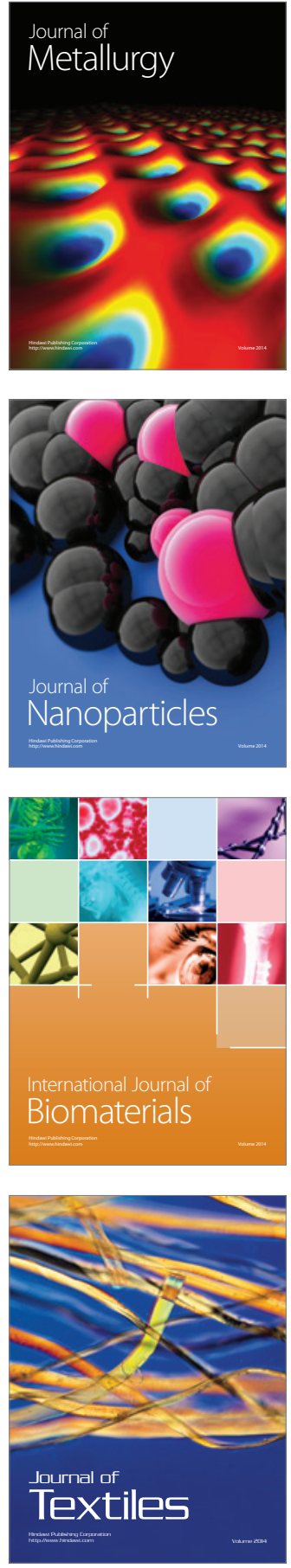